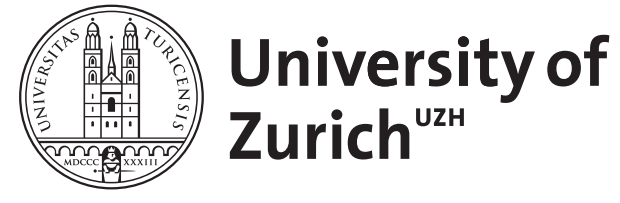

\title{
Simulation des Blutflusses in der abdominellen Aorta
}

\author{
Frauenfelder, T ; Boutsianis, E ; Alkadhi, H ; Marincek, B ; Schertler, T
}

\begin{abstract}
Das Ziel der numerischen Simulation des Blutflusses in der Aorta ist, die Mechanik der Entstehung von Aortenaneurysmen im Hinblick auf das Rupturrisiko zu untersuchen und die Wirkungen interventioneller Maßnahmen zu beschreiben. Die Grundlage der numerischen Simulation sind virtuelle Modelle von Gefäßen und die physikalischen Eigenschaften der Gefäßbestandteile, des Blutes und der Strömung. Basierend auf diesen Angaben werden mit Hilfe numerischer Methoden die strömungsmechanischen Probleme des Blutflusses näherungsweise gelöst. Die Ergebnisse können dann quantitativ und qualitativ dargestellt werden. Die Ergebnisse der numerischen Flusssimulation zeigen, dass in abdominellen Aortenaneurysmen die Höhe des Wanddrucks, der von entscheidender Bedeutung für das Rupturrisiko ist, von verschiedenen Faktoren, wie z.B. der Lage des Wandthrombus, abhängt. In Modellen mit Stentgrafts wurden mit Hilfe der numerischen Simulation Faktoren, welche die Stentgraftmigration beeinflussen, untersucht. Obwohl die numerische Simulation des Blutflusses noch einige Limitationen aufweist, zeigen aktuelle Studien, dass die Methode das Potenzial hat, um in Zukunft eine dedizierte Beurteilung des Rupturrisikos von Aortenaneurysmen vorzunehmen
\end{abstract}

DOI: https://doi.org/10.1007/s00117-007-1576-7

Posted at the Zurich Open Repository and Archive, University of Zurich ZORA URL: https://doi.org/10.5167/uzh-156579

Journal Article

Published Version

Originally published at:

Frauenfelder, T; Boutsianis, E; Alkadhi, H; Marincek, B; Schertler, T (2007). Simulation des Blutflusses in der abdominellen Aorta. Der Radiologe, 47(11):1021-1028.

DOI: https://doi.org/10.1007/s00117-007-1576-7 
Radiologe 2007 · 47:1021-1028

DOI 10.1007/s00117-007-1576-7

Online publiziert: 4. November 2007

(c) Springer Medizin Verlag 2007
T. Frauenfelder ${ }^{1} \cdot$ E. Boutsianis ${ }^{2} \cdot$ H. Alkadhi ${ }^{1} \cdot$ B. Marincek ${ }^{1} \cdot$ T. Schertler $^{1}$

${ }^{1}$ Institut für Diagnostische Radiologie, Universitätsspital Zürich

${ }^{2}$ Labor für Computerwissenschaften und Engineering, ETH, Zürich

\section{plus Video}

Dieser Beitrag enthält anschauliches Videomaterial. Bitte folgen Sie diesem Pfad: www.DerRadiologe.de $\rightarrow$ Online-Archiv $\rightarrow$ Beitrag $\rightarrow$ Supplemental Material

\begin{abstract}
Kenntnisse über die Entstehung von Aortenaneurysmen sind von entscheidender Bedeutung für die Einschätzung des Rupturrisikos. Diverse Studien auf molekularer Basis haben gezeigt, dass die Strömungseigenschaften des Blutes und die daraus resultierenden, auf die Gefäßwand wirkenden Kräfte, einen entscheidenden Einfluss auf die Entstehung der Arteriosklerose und die Aneurysmabildung haben. Die numerische Simulation, basierend auf In-vivo-Daten, bietet die Möglichkeit, den Blutfluss und seine Auswirkungen auf die Gefäßwände in anatomisch korrekten Gefäßmodellen darzustellen.
\end{abstract}

Die numerische Simulation des Blutflusses in der Aorta wurde v. a. von Forschern der Biomechanik vorangetrieben. Sie hatte zum Ziel, die Mechanik der Aortenaneurysmaentstehung im Hinblick auf das Rupturrisiko zu untersuchen und die Wirkungen interventioneller Maßnahmen zu beschreiben. Das abdominelle Aortenaneurysma ist eine degenerative Erkrankung der Aorta, die zu einer Erweiterung des Lumens und zur Schwächung der Arterienwand führt, die bei fehlender Therapie reißen kann. Gestützt auf die Definition, dass eine Erweiterung der infrarenalen Aorta auf mehr als $29 \mathrm{~mm}$ ein Aneurysma ist, haben $8,8 \%$ der Bevölkerung

\section{Simulation des Blutflusses in der abdominellen Aorta}

\section{Die numerische Simulation des Blutflusses in abdominellen Aortenaneurysmen vor und nach Intervention}

über 65 Jahre ein abdominales Aortenaneurysma $[1,2]$.

Die Entscheidung für eine elektive chirurgische bzw. interventionelle Therapie oder ein Abwarten mit periodischen Kontrollen ist schwierig, da sichere Anhaltspunkte zur Bestimmung des Rupturrisikos fehlen [3]. Die im klinischen Alltag verwendeten Indikationen zur Intervention (Erreichen eines maximalen transversalen Durchmessers von mehr als 5 bzw. $6 \mathrm{~cm}$ ) basiert auf einzelnen Verlaufsstudien [4]. Es bestehen aber klinische Hinweise, dass auch kleine Aneurysmen plötzlich mit einer perioperativen Mortalität von über 50\% rupturieren können $[5,6]$. Dementsprechend sollte möglichst die interventionelle Korrektur des Aortenaneurysmas nicht allein in Abhängigkeit des Maximaldurchmessers erfolgen, sondern auch anhand zuverlässigerer Parameter zur Abschätzung des Rupturrisikos.

Aus der Sicht der Mechanik rupturiert das Aneurysma, wenn die Kraft, die auf die Wand wirkt, größer ist als die Festigkeit des Wandgewebes. Diese Kräfte lassen sich aber in vivo nicht messen. Die Wandbelastung wird von einer Vielzahl von Faktoren, wie zum Beispiel Durchmesser und Form des Aneurysmas, Charakteristiken der Wand, Form und Lage eines etwaigen intraluminalen Thrombus, und deren Interaktionen beeinflusst [3].

Zur Darstellung des Zusammenspiels dieser verschiedenen Komponenten wur- de in den letzten Jahrzehnten vermehrt die numerische Flusssimulation benutzt. Ziel ist es einerseits, Vorgänge, die zur Schwächung der Gefäßwand führen, zu erforschen, um in Zukunft das Rupturrisiko besser abschätzen zu können, und anderseits die Wirkung therapeutischer Maßnahmen darzustellen.

Verschiedene experimentelle und numerische Studien haben den Blutfluss und dessen Auswirkungen auf die aortale Gefäßwand vor und nach Einlage eines Stentgrafts erforscht und die Verteilung der Kräfte interpretiert. Fillinger et al. [7] berechneten die maximalen Wandbelastungen in anatomisch korrekten Modellen von Aneurysmen. Di Martino et al. [3] und Finol et al. [8] analysierten, welche systemischen Parameter für die Beurteilung des Rupturrisikos von Bedeutung sind. Andere Gruppen untersuchten die Verteilung der Wandscherkräfte von $3 \mathrm{D}$ rekonstruierten Modellen [9].

Eine große Zahl an Studien simulierte den Blutfluss in Stentgrafts und stellte die hämodynamischen Auswirkung der Intervention dar $[10,11,12,13,14]$.

\section{Was ist numerische Flusssimulation?}

Die numerische Flusssimulation (engl. "computational fluid dynamics", CFD) ist eine etablierte Methode der Strömungsmechanik. Sie hat zum Ziel, strömungs- 

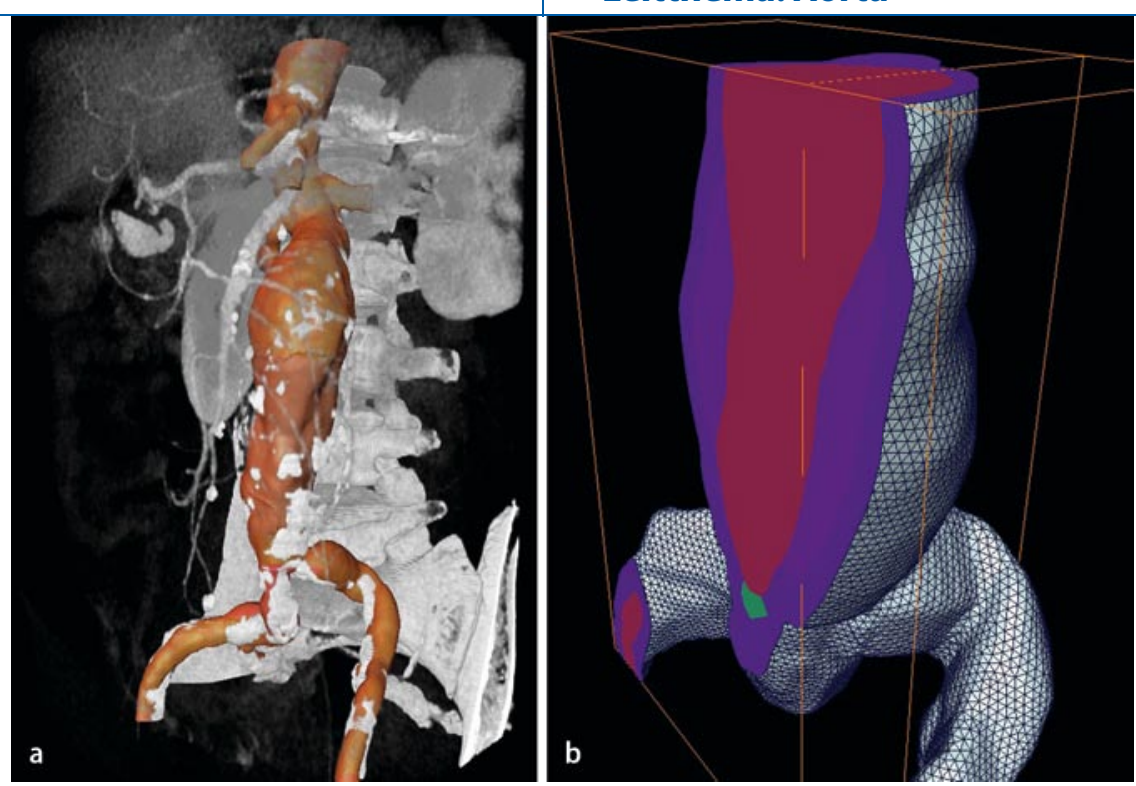

Abb. $1 \Delta$ a Darstellung eines anatomisch korrekten Modells eines Aortenaneurysmas. b Schnitt durch ein Modell mit Darstellung der einzelnen Materialien und der Gitternetzstruktur

mechanische Probleme näherungsweise mit numerischen Methoden zu lösen. Die Grundgleichungen der Strömungsmechanik sind die Navier-Stokes-Gleichungen, welche die Strömungsgeschwindigkeit und Druckverteilung in Newton-Flüssigkeiten und Gasen beschreiben [15].

Die Navier-Stokes-Gleichungen stellen ein System nichtlinearer partieller Differenzialgleichungen 2. Ordnung dar. Der Kern dieser Gleichungen ist der Impulssatz. Je nach Art des betrachteten Fluidums müssen zusätzlich der Massen- und der Energieerhaltungssatz zur Vervollständigung der Gleichungen angewendet werden.

Unter Newton-Fluiden versteht man Flüssigkeiten oder Gase, deren Scherspannung proportional zur Schergeschwindigkeit ist. Dies trifft für Wasser, Luft und verschiedene Öle zu, jedoch nicht für Blut. Die komplexe Zusammensetzung des Blutes aus Plasma und deformierbaren und adhäsiven Blutzellen beeinflusst entscheidend die Viskosität. Somit müsste Blut streng genommen als eine Nicht-Newton-Flüssigkeit betrachtet werden, was jedoch aufgrund des geringen Einflusses der Zellen in großen Gefäßen oft bewusst vernachlässigt wird, da die Ergebnisse nicht signifikant abweichen.

$\mathrm{Zu}$ den meist genutzten Lösungsmethoden der numerischen Strömungsgleichungen zählt die Finite-Elemente-Methode, die sich für viele Problemstellun- gen mit nicht komprimierbaren Fluiden eignet. Hierbei wird das Modell, das für die Simulation bzw. Strömungsberechnung gewählt wurde, in eine große Zahl kleiner, aber endlich vieler Elemente unterteilt. In anatomisch korrekten $3 \mathrm{D}-\mathrm{Mo}-$ dellen sind diese Elemente meistens Tetraeder. Für diese Elemente werden Initialwerte definiert, d. h. Werte an den Einund Ausflüssen, mit denen die Simulation durchgeführt werden soll. Diese Initialwerte können Flussgeschwindigkeiten $(\mathrm{m} / \mathrm{s})$, Druckveränderungen $(\mathrm{Pa} / \mathrm{s})$ oder Massenflüsse ( $\mathrm{ml} / \mathrm{s})$ sein. Das daraus resultierende Gleichungssystem setzt sich aus den Differenzialgleichungen und den vom Anwender gewählten Materialeigenschaften und Flussbedingungen zusammen. Zudem werden die Beziehungen zwischen Wand und Fluidum definiert.

Die Strömung der Flüssigkeit kann als laminar oder turbulent klassifiziert werden. Laminar bedeutet, dass sie in Schichten strömt, die sich nicht vermischen. Im Gegensatz dazu ist die turbulente Strömung die Bewegung von Flüssigkeiten und Gasen, bei der Verwirbelungen auftreten. Der Übertritt von der laminaren zur turbulenten Strömung erfolgt ab einer bestimmten Strömungsgeschwindigkeit. Als Kriterium wird hierzu die Reynolds-Zahl (Re) verwendet [15]. Dieser Wert liegt zum Beispiel für die Röhrenströmung bei etwa 2300 Re.

\section{Praktisches Vorgehen}

Die praktische Vorgehensweise zur Durchführung der numerischen Flusssimulation in der Aorta, basierend auf der Finite-Elemente-Methode, gliedert sich in mehrere Abschnitte, die

- Modellerstellung,

- Einstellung und Definition der Materialgrenzen und der Anfangsbedingungen („preprocessing“),

- eigentliche Simulation, bzw. Lösung der Navier-Stokes-Gleichungen (Simulation),

- Nachverarbeitung („postprocessing“).

\section{Modellerstellung}

Parallel zur raschen Entwicklung der Informatiktechnologie mit immer leistungsfähigeren Prozessoren nahm auch die Komplexität der Modelle zu, da es nun möglich war, komplexe Modelle in gleicher oder kürzerer Zeit zu simulieren. Erste Simulationen wurden an stark vereinfachten $2 \mathrm{D}$-Abbildungen von Röhren mit Ausbuchtungen vorgenommen. Im weiteren Verlauf ging man dazu über, Strömungen in 3 D-Röhren mit Aufzweigungen zu simulieren [12]. Diese Modelle stellen stark vereinfachte Geometrien der Gefäße dar.

Erst in den letzten Jahren ist man dazu übergegangen, $3 \mathrm{D}$-Modelle gestützt auf Magnetresonanz- bzw. Computertomographiedaten von Patienten zu erstellen und damit reale Verhältnisse zu simulieren $[3,14,16]$.

Die Erstellung des Modells beginnt mit der semiautomatischen Segmentierung der gewünschten Gefäßanteile wie der Wand, des Wandthrombus und größerer Verkalkungen sowie - in interventionellen Fällen - des Stentgrafts. Aus den segmentierten Daten wird mit Hilfe des sog. General-marching-cube-Algorithmus ein $3 \mathrm{D}$-Volumendatensatz erstellt [17]. Das sichtbare patientenspezifische Modell, bestehend aus einem Oberflächennetz unterschiedlich großer und unterschiedlich geformter Dreiecke, bildet die Grundlage der numerischen Simulation (• Abb. 1a). Dieses Oberflächennetz wird mittels spezieller Programme (Gitternetzgeneratoren, z. B. Gambit, Fluent Corp., Darmstadt, Deutschland) in ein 
möglichst gleichförmig strukturiertes Volumengitternetz aus in etwa gleich großen und gleich geformten Tetraedern transformiert, wobei zusätzlich die verschiedenen Grenzflächen zwischen den Materialien und die Ein- und Ausflüsse definiert werden (- Abb. 1b). Ein solches Volumennetz besteht aus ca. 500.000-1.000.000 Tetraedern.

\section{Simulationsvorbereitung (Preprocessing)}

Die Simulationsvorbereitung dient der Definition der verschiedenen Parameter für die Eigenschaften der Grenzflächen und Materialien sowie der Festlegung der Initialwerte. Die Definition der Materialeigenschaften erfolgt durch Festlegung von Viskosität, Dichte, spezifischem Gewicht, Young-Modulus und PoissonKoeffizient für die verschiedenen $\mathrm{Ma}$ terialien wie Blut, Thrombus, Stent und Wand. Der Young-Modulus oder Elastizitätsmodul ist ein Maß für die Elastizität des Modells. Der Wert des Elastizitätsmoduls ist umso größer, je mehr Widerstand ein Material seiner Verformung entgegensetzt, d. h. je höher der Wert desto steifer das Material. Der Poisson-Koeffizient ist das Verhältnis aus relativer Änderung der Dicke zu relativer Änderung der Länge bei Einwirkung einer äußeren Kraft oder Spannung. Der Koeffizient beruht auf dem Phänomen, dass elastische Materialien, die in einer Richtung verlängert werden, gleichzeitig senkrecht dazu verkürzt werden.

Die Grenzflächen an den Gefäßwänden werden meistens als rutschfest definiert, d. h. die Flussgeschwindigkeit entlang der Wand ist null.

An den Ein- oder Ausflüssen werden die Initialwerte (z. B. Flussgeschwindigkeit) definiert. Die Werte können konstant (gleiche Werte über eine gewisse Zeit), linear (gleiche Veränderung der Werte über die Zeit), sinusoidal oder pulsatil (ständige Veränderung der Werte über die Zeit) sein. Die Materialeigenschaften des Fluidums, der Materialien und die Initialwerte definieren die Strömungseigenschaften. Für Flusssimulationen in der Aorta wird zumeist eine laminare Strömung einer nichtkomprimierbaren Flüssigkeit angenommen.

Radiologe 2007· 47:1021-1028 DOI 10.1007/s00117-007-1576-7

(c) Springer Medizin Verlag 2007

T. Frauenfelder · E. Boutsianis · H. Alkadhi · B. Marincek · T. Schertler Simulation des Blutflusses in der abdominellen Aorta. Die numerische Simulation des Blutflusses in abdominellen Aortenaneurysmen vor und nach Intervention

Zusammenfassung

Das Ziel der numerischen Simulation des Blutflusses in der Aorta ist, die Mechanik der Entstehung von Aortenaneurysmen im Hinblick auf das Rupturrisiko zu untersuchen und die Wirkungen interventioneller Maßnahmen zu beschreiben. Die Grundlage der numerischen Simulation sind virtuelle Modelle von Gefäßen und die physikalischen Eigenschaften der Gefäßbestandteile, des Blutes und der Strömung. Basierend auf diesen Angaben werden mit Hilfe numerischer Methoden die strömungsmechanischen Probleme des Blutflusses näherungsweise gelöst. Die Ergebnisse können dann quantitativ und qualitativ dargestellt werden.

Die Ergebnisse der numerischen Flusssimulation zeigen, dass in abdominellen Aortenaneurysmen die Höhe des Wanddrucks, der von entscheidender Bedeutung für das
Rupturrisiko ist, von verschiedenen Faktoren, wie z. B. der Lage des Wandthrombus, abhängt.

In Modellen mit Stentgrafts wurden mit Hilfe der numerischen Simulation Faktoren, welche die Stentgraftmigration beeinflussen, untersucht. Obwohl die numerische $\mathrm{Si}$ mulation des Blutflusses noch einige Limitationen aufweist, zeigen aktuelle Studien, dass die Methode das Potenzial hat, um in Zukunft eine dedizierte Beurteilung des Rupturrisikos von Aortenaneurysmen vorzunehmen.

\section{Schlüsselwörter}

Computergestützte numerische Analyse . Computersimulation · Aorta abdominalis Aortenaneurysma $\cdot$ Stents

\section{Simulation of blood flow within the abdominal aorta. Computational fluid dynamics in abdominal aortic aneurysms before and after interventions}

\section{Abstract}

The goal of numeric analysis of aortic blood flow is to evaluate the mechanisms leading to an aortic aneurysm with regard to the risk of a rupture and to describe the effect of interventional therapy. Numeric analysis is based on virtual models of vascular structures and the physical characteristics of the vessel wall, of blood as fluidum, and the blood flow. Using this information, numeric analysis solves the appropriate equations. The results can be displayed quantitatively and qualitatively.

The results of numeric flow simulation show that in abdominal aortic aneurysms the wall pressure, which is of vital importance for the risk of rupture, depends on several fac- tors, one being the location of the intraluminal thrombus.

In models of aneurysms after stent grafting, numeric analysis can be used to evaluate factors leading to stent migration. Although numeric analysis of aortic blood flow still has several limitations, recent studies have shown that this method has the potential for improved estimation of the rupture risk of aortic aneurysms in the near future.

\section{Keywords}

Computer-assisted numeric analysis . Computer simulation - Aorta abdominal . Aortic aneurysm $\cdot$ Stents 


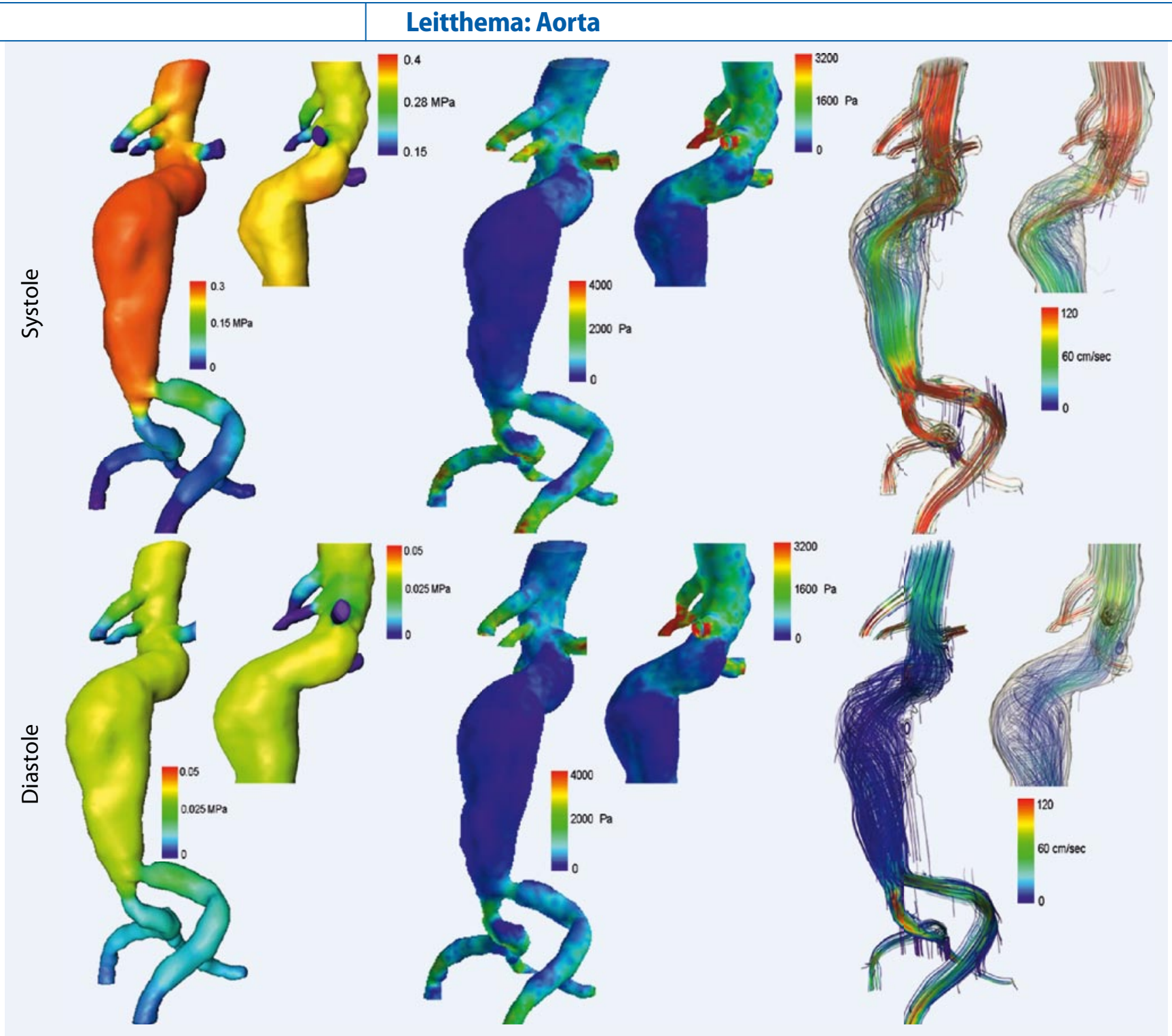

Abb. $2 \Delta$ Darstellung des Wanddrucks, der Wandscherkraft und des Strömungsmusters eines abdominellen Aortenaneurysmas während der Systole und Diastole

\section{Simulation}

Zur Lösung der Navier-Stokes-Gleichungen mit den vorher festgelegten $\mathrm{Pa}$ rametern wird ein spezifischer Lösungscode verwendet. Dieser Code basiert z. B. auf der Finite-Elemente-Methode. Durch die zusätzliche Wahl eines sog. FluidStruktur-Interaktion-Ansatzes ist es möglich, simultan die mechanischen Vorgänge in der Flüssigkeit und Struktur (Wand, Stent) zu simulieren. Das Programm löst hierzu die Gleichungen für Flüssigkeitsund Strukturveränderungen und berechnet zusätzlich die Veränderungen im Gitternetz. Dadurch kann z. B. die Wandbewegung simuliert werden [14]. Bei nicht konstanten Eingabewerten werden die- se Gleichungen für jeden Zeitabschnitt in Abhängigkeit vom vorangegangenen Zeitabschnitt berechnet.

Die Dauer zur Lösung der Gleichungen ist von der Komplexität des Modells sowie der Anzahl der Elemente abhängig. Die Zahl der Elemente muss genügend hoch gewählt werden, sodass die erhaltene Lösung unabhängig von der Gitternetzstruktur ist.

\section{Nachverarbeitung (Postprocessing)}

Zur Nachverarbeitung der Ergebnisse stehen verschiedene Visualisierungs- und Quantifizierungsprogramme zur Verfügung. Diese ermöglichen eine detaillierte qualitative und quantitative Auswertung der Simulationsergebnisse.

\section{Flusssimulation in abdominellen Aortenaneurysmen vor Intervention}

Das Hauptziel der numerischen Simulation des Blutflusses in Aortenaneursymen ist es, die hämodynamischen Veränderungen zu beschreiben und Faktoren zu erforschen, die eine bessere Abschätzung des Rupturrisikos ermöglichen ( $\bullet$ Abb. 2, 3). Der erste Faktor, der mit dem Rupturrisiko in Verbindung gebracht wurde, ist der intraluminale Druck gegen die Wand. Vor allem der senkrecht auf die Wand einwirkende Druck wurde als einer der wich- 
tigsten Faktoren angesehen und in verschiedenen Studien untersucht $[3,9,18]$. Um ein möglichst genaues Resultat zu erhalten, wurden große Anstrengungen bei der Modellierung physikalischer Eigenschaften der virtuellen abdominellen Aortenaneurysmen unternommen. Während früher vereinfachte Modelle von Aneurysmen zur Berechnung der Wandbelastungen verwendet wurden [8], werden heute vermehrt anatomisch korrekte Modelle, basierend auf CT- oder MR-Datensätzen, eingesetzt $[3,14,16,19]$.

Vom biomechanischen Standpunkt aus rupturiert die Aorta, wenn der intraluminale Druck die Festigkeit der erkrankten Aortenwand übersteigt. In 2 Arbeiten wurde diese Kraft an resezierten $\mathrm{Ge}$ fäßen gemessen und betrug ca. $65 \mathrm{~N} / \mathrm{cm}^{2}$ für Aortenaneurysmen [3, 9]. Dieser Wert liegt höher als der von Fillinger et al. [7] gemessene Wert von $47,7 \pm 6 \mathrm{~N} / \mathrm{cm}^{2}$ für simulierte Modelle von Patienten mit einer Aortenruptur, wobei diese Werte nur für die in dieser Studie verwendeten Simulationsbedingungen galten. Aktuelle Studien zeigen, dass das biomechanische Umfeld, in dem der Wanddruck erzeugt wird, von diversen anderen Faktoren wie der Form des Aneurysmas, dem Vorhandensein eines Wandthrombus oder der Wanddicke abhängt $[3,7,18,19]$.

Doch auch biologische Veränderungen korrelieren mit der Strömung. So konnte gezeigt werden, dass die Scherkraft eng mit der intimalen Hyperplasie in der Aorta korreliert [20]. Für das Rupturrisiko spielte die Scherkraft bisher eine untergeordnete Rolle, da sie ca. 4 Potenzen kleiner ist als der senkrecht auf die Wand wirkende Druck. Eine neue Arbeit zeigte jedoch, dass hohe Scherkraft- und Druckgradienten an derselben Stelle wie die Druckmaxima auftreten ( Abb. 2). Somit muss ein Einfluss dieser Parameter als wahrscheinlich angesehen werden. In der gleichen Arbeit wurde auch gezeigt, dass die Lokalisation des maximalen Wanddrucks nicht mit dem maximalen Durchmesser korreliert, und dass der Wanddruck nicht unbedingt während der Systole am höchsten ist [19].

Zusätzlich zur Hämodynamik wird dem intraluminalen Thrombus eine wichtige Bedeutung zugemessen. Einerseits beeinflusst die Strömung die Bildung und

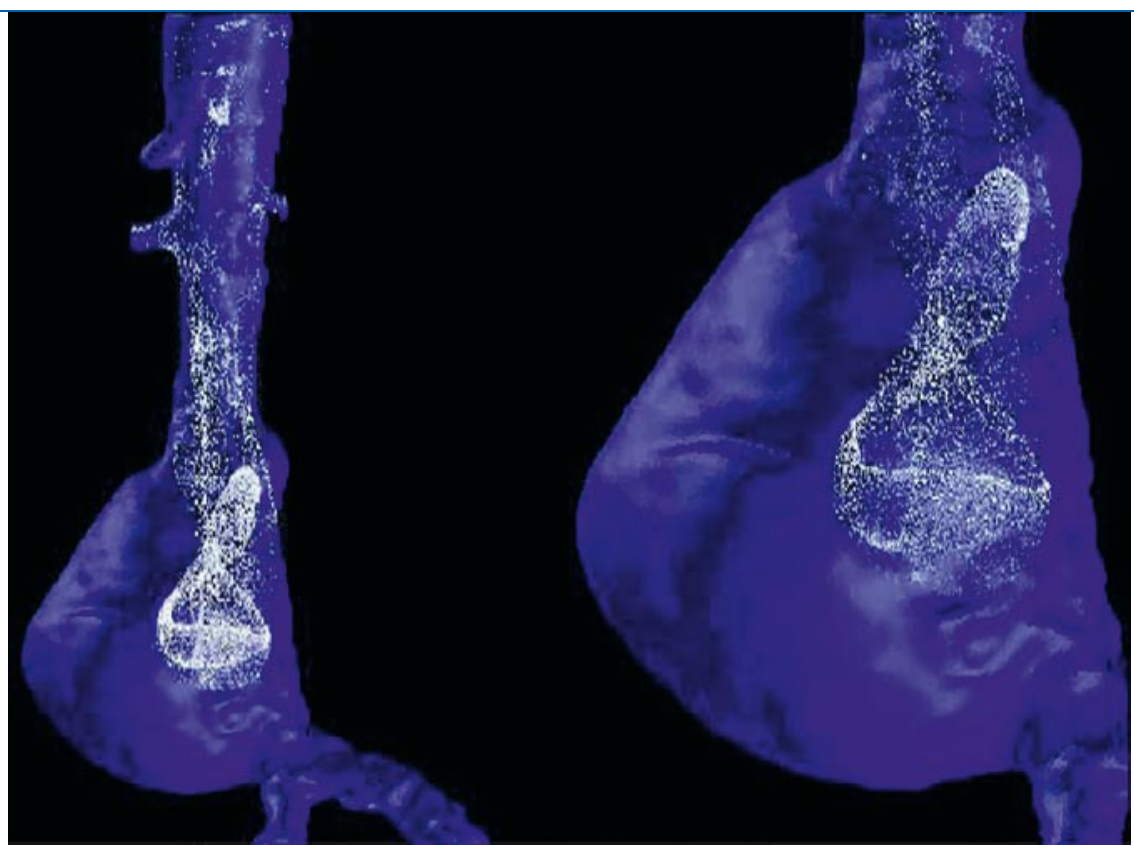

Abb. $3 \Delta$ Pulsatiler Blutfluss in einem abdominellen Aortenaneurysma, dargestellt mittels Partikeln (Einzelbild aus dem Videofilm)

Stabilisierung des intraluminalen Thrombus, andererseits verändert sich die Strömung durch die Thrombenbildung. In Autopsien rupturierter Aorten wurde der Thrombus an der Stelle der Ruptur oder in unmittelbarer Nachbarschaft gefunden [21]. Neue Studien zeigen, dass der intraluminale Thrombus die Wand vor dem Einfluss der Druckgradienten schützt [22]. So liegen die maximalen Druckgradienten an Lokalisationen ohne oder mit nur sehr dünnem Thrombusmaterial [19].

\section{Flusssimulation in abdominellen Aortenaneurysmen nach Intervention}

Die numerische Flusssimulation bei Patienten nach Stentgraftimplantation befasst sich v. a. mit der Problematik der Stentmigration und den Auswirkungen von Endoleaks auf den Stent ( $\bullet$ Abb. 4, 5). Es wird angenommen, dass die Ursache eine ungenügende Fixation oder eine Zunahme des Durchmessers der Aorta am Stenthals ist. Zarins et al. [23] berichteten über eine Stentgraftmigrationsrate von $18,8 \%$ in einer Population von 1119 Patienten nach der 3. Jahreskontrolle.

Migration entsteht, wenn die Zugkraft am Stent die proximale Fixationskraft übersteigt. Wichtige Faktoren, die den Stentgraft fixieren, sind die Reibung, die angebrachten Haken, ein langer Aneurysmahals, die suprarenale Fixation und das Einwachsen in die Wand. Resch et al. [24] untersuchten die proximalen Stentfixationskräfte und maßen Werte zwischen $4,5 \mathrm{~N}$ für einen Talent- und $24 \mathrm{~N}$ für einen Zenith-Stent. Die Zugkraft am Stent entsteht v. a. durch den Druck, der auf den Stent im Bereich der Bifuraktion wirkt. Faktoren, welche die Zugkraft am Stentgraft beeinflussen, sind der systemische Blutdruck, der proximale Stentdurchmesser, die Angulation des Halses sowie der Winkel zwischen den Schenkeln.

Eine aktuelle Studie deutet darauf hin, dass eine Überdimensionierung des Stents im proximalen Fixationspunkt das Migrationsrisiko erhöht [25]. Diese Arbeit wird durch eine klinische Arbeit über ZenithStents unterstützt [26]. Allgemein wird der Körper des Stents größer gewählt, um eine gute Fixation zu erreichen. Durch den Wegfall des Drucks im Aneursymasack kommt es zumeist zu einer Erweiterung des Gefässhalses bis zum maximal möglichen Durchmesser des Stents. Da nun der Einflussdurchmesser einen entscheidenden Einfluss auf die Zugkraft hat, führt ein zu groß gewählter Stent eher zu einer Migration [25]. Diese Zugkraft ist zudem höher bei Patienten mit Hypertonie. 


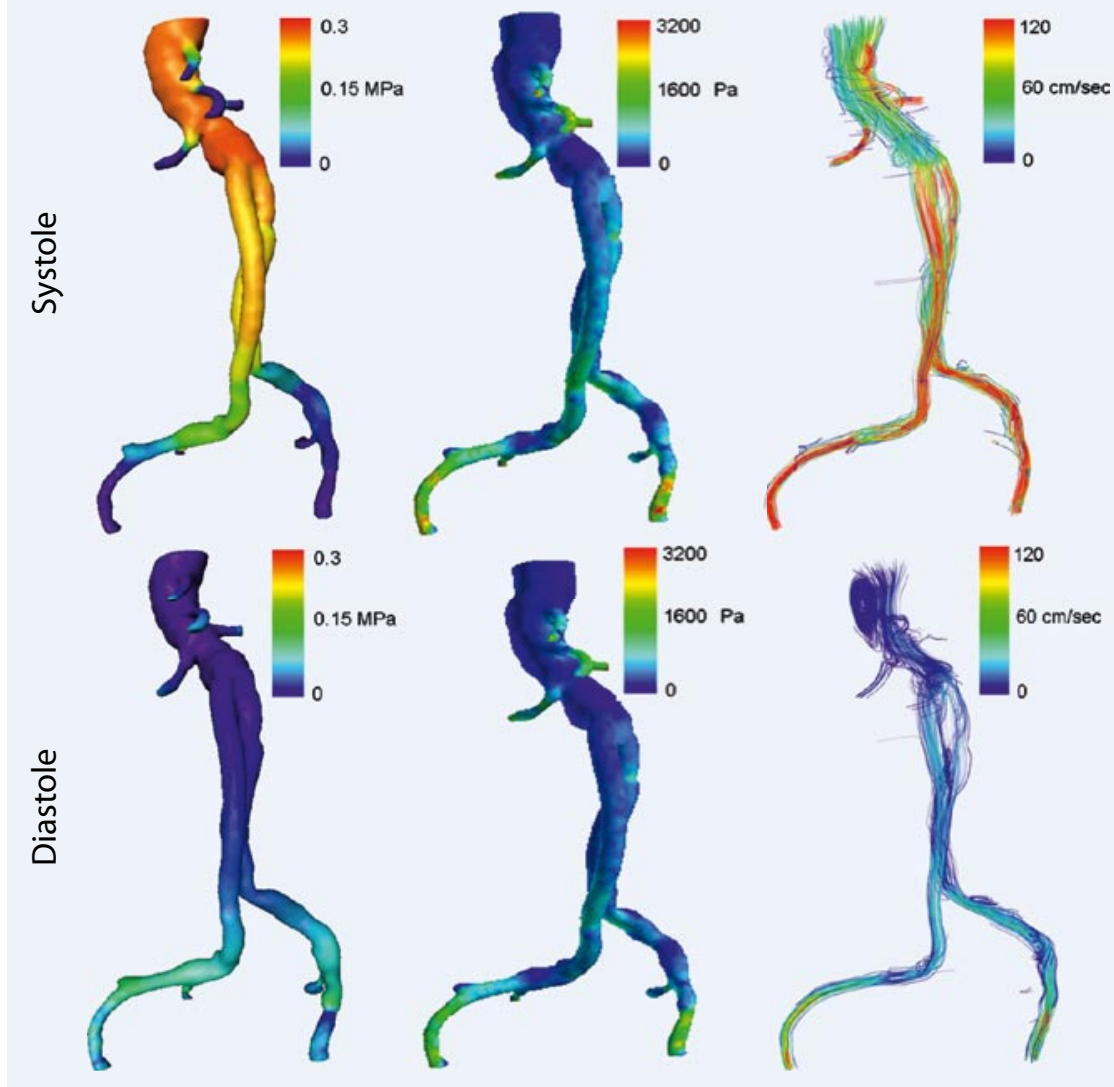

Abb. $4 \Delta$ Darstellung des Wanddrucks, der Wandscherkraft und des Strömungsmusters eines mittels Endoprothese versorgten abdominellen Aortenaneurysmas während der Systole und Diastole

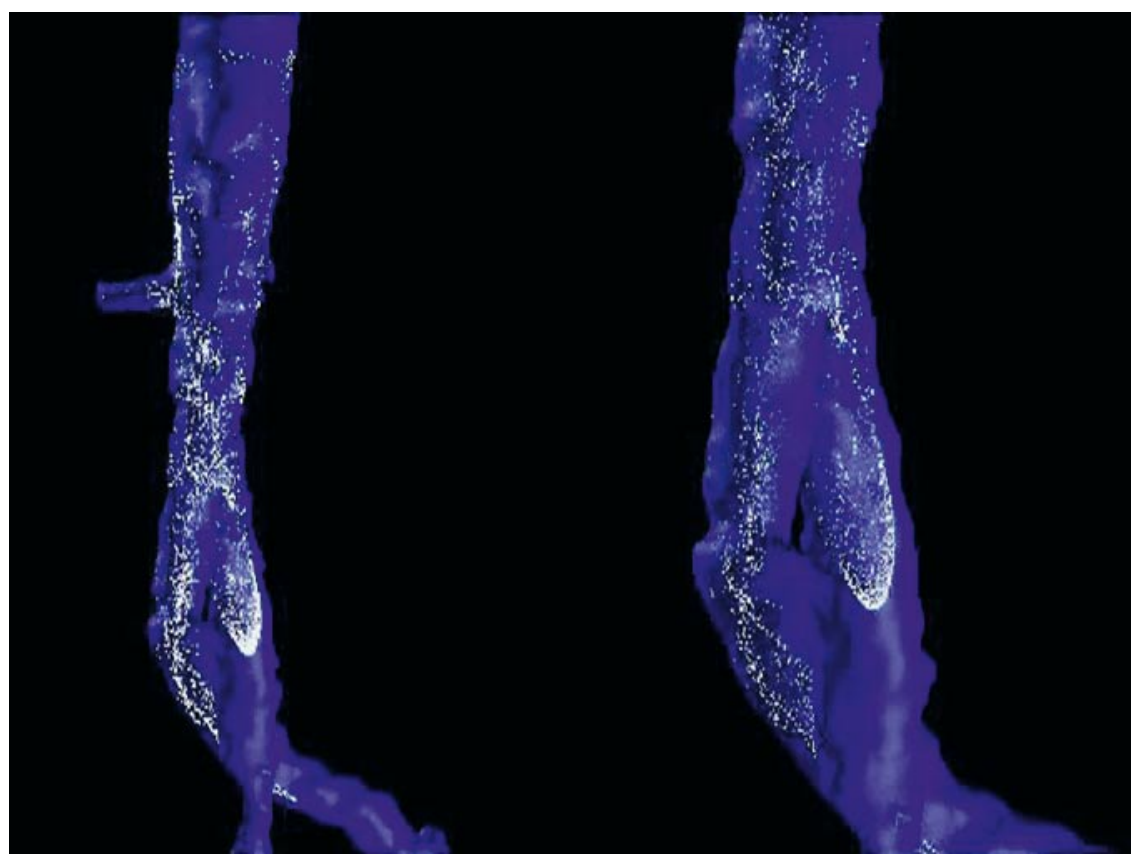

Abb. $5 \Delta$ Pulsatiler Blutfluss in einem mittels Endoprothese versorgten abdominellen Aortenaneurysma, dargestellt mittels Partikeln (Einzelbild aus dem Videofilm)
Neben dem maximalen Durchmesser des Stentkörpers haben auch die Länge des Stentkörpers und der Stentschenkel einen entscheidenden Einfluss auf die Zugkraft (- Abb. 6) So sind kurze Schenkel in der Lage, den Druck direkt an die Beckengefäße weiterzuleiten, während es in langen Schenkeln zu einem starken Druckabfall im proximalen Anteil mit stark erhöhten Druckwerten im angedockten Stentschenkel kommt [14, 27]. Nicht ohne Grund wird diese Stelle oft auch als vulnerable Stelle oder „weak spot“ bezeichnet [27]. Die Flusssimulation mit Einbezug der Flüssigkeits-Struktur-Interaktion konnte zeigen, dass die Einlage des Stentgrafts zu einer Abnahme des Drucks im Aneurysmasack im Mittel um das 10- bis maximal 20-fache während der Systole führt [10].

Innerhalb des Aneurysmasacks weist der Druck eine pulssynchrone Variation auf. Diese ist durch komplexe Strukturinteraktionen zwischen Blutfluss, Stentwand, stagnierendem Blut und Thrombus bedingt. Liegt ein Endoleak Typ II vor, konnten Li u. Kleinstreuer [28] zeigen, dass der Druck im zuführenden Gefäß direkt an den Aneurysmasack weitergeleitet wird und es zu einem Druckanstieg im Aneurysmasack kommt. Dies führt zu einer deutlichen Abnahme der Zugkraft am Stentgraft, da diese von der Druckdifferenz zwischen Stentgraft und Aneurysmasack abhängt. Liegt nun der Druck im zuführenden Gefäß über dem halben Systemdruck, wird nicht nur die Gefahr einer Migration gebannt, sondern auch die Gefahr der Bildung eines Endoleaks Typ I. Andererseits ist aufgrund des konsekutiven Wanddruckanstiegs mit einer erneuten Zunahme des Rupturrisikos zu rechnen.

\section{Validierung der Flusssimulation}

Die Überprüfung der Genauigkeit der Flusssimulation hat in 2 Punkten zu erfolgen: Einerseits muss das Ergebnis unabhängig von der Gitternetzstruktur sein, andererseits müssen die Strömungsgeschwindigkeit und das Strömungsmuster mit den tatsächlichen Werten übereinstimmen.

Die Genauigkeit der Simulation hängt vom Feinheitsgrad des Gitternetzes bzw. 


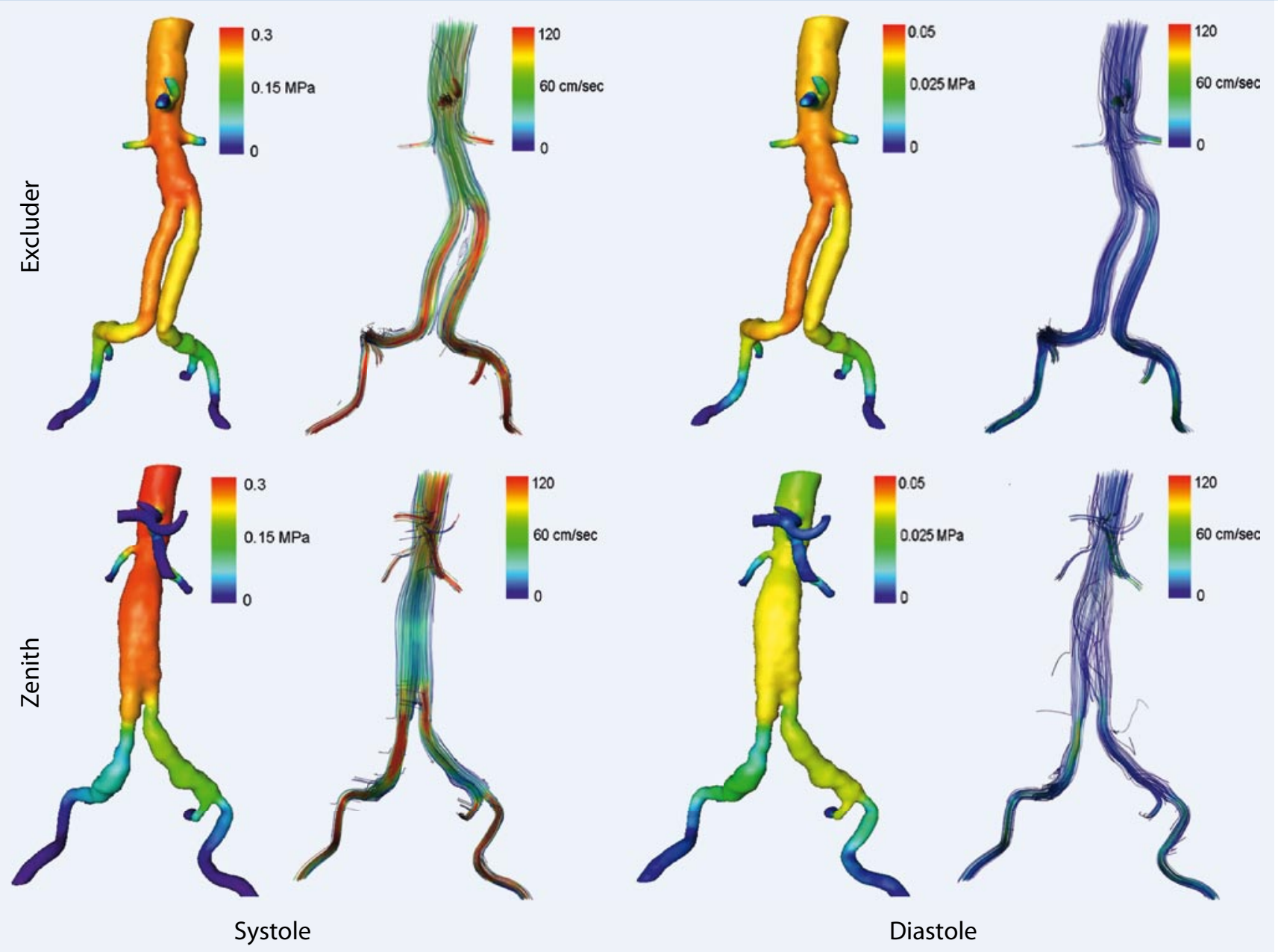

Abb. $6 \Delta$ Vergleich des Drucks und des Strömungsmusters zwischen einem Excluder- und einem Zenith-Stentgraft während der Systole und Diastole

der Anzahl der Elemente ab. Es besteht ein Schwellenwert, oberhalb dessen keine signifikant besseren Ergebnisse erzielt werden. Ziel muss es sein, diesen Schwellenwert zu erkennen, da jede höhere Zahl der Elemente die Berechnungszeit stark verlängert (bis hin zu Monaten). Durch den Vergleich der Simulationsergebnisse verschiedener Dichtegrade lässt sich dieser Schwellenwert ableiten. In der von uns vorgestellten Arbeit lag dieser Wert bei ca. 6oo.ooo Elementen [14].

Zur Überprüfung der Genauigkeit im Vergleich zum realen Fluss werden die Strömungsmuster und -geschwindigkeiten der numerischen Flusssimulation mit Werten, die in Glas- oder Silikonmodellen gemessen werden, verglichen, da ein direkter Vergleich mit In-vivo-Modellen zurzeit nicht möglich ist. Diese „experimentellen“ Flusssimulationen bedürften einer aufwändigen Infrastruktur, bestehend aus Pumpen, realistischen Mo- dellen und Messverfahren mit Laseroptiken [14]. Ergebnisse solcher Vergleichsstudien konnten eine hohe Übereinstimmung der Flussmuster und -geschwindigkeiten zeigen $[14,29]$.

\section{Limitationen der Methode}

Wie oben erwähnt, können reale Situationen zurzeit nicht präzise simuliert werden. So ist Blut ist eine sehr komplizierte Suspension, deren Zellen einen zusätzlichen Einfluss auf die Gefäßwand haben. Auch die Gefäßwand, mit den verschiedenen Wandschichten und der bekannten zellulären Kommunikation bzw. Reaktion auf Scherkräfte, kann in den Simulationen nur vereinfacht dargestellt werden. Obwohl durch verbesserte Bildgebungsmethoden detailgetreuere Informationen der Gefäße zu erhalten sind, ist es derzeit nicht möglich, alle Faktoren, die bei der Gefäßerkrankung eine Rolle spielen, in die numerische Flusssimulation einzuschließen. Bereits eine höhere Auflösung der Wand mit einer höheren Anzahl an Elementen und das Verwenden mehrerer Materialien würden die Rechenkapazitäten eines Hochleistungsrechners für Monate auslasten.

Des Weiteren können viele für eine exakte Simulation relevante patientenspezifische Informationen, wie z. B. die Gefäßwandfestigkeit, die Druckverhältnisse im Retroperitoneum, die Strömungsverhältnisse in den vor- und nachgeschalteten Gefäßen und die Eigenschaften des Wandthrombus in vivo nicht bestimmt werden.

\section{Fazit für die Praxis}

Die numerische Flusssimulation ist eine viel versprechende Methode zur Berechnung und Darstellung des Blutflusses in der Aorta mitsamt dessen Auswirkungen 
auf Wanddruck und Scherkräfte. Diese Informationen haben das Potenzial, eine zukünftig bessere Prognose des Rupturrisikos der Aorta zu erlauben und den Nutzen und etwaige Komplikationen von Interventionen zu illustrieren. Aufgrund der oben geschilderten Limitationen ist eine routinemäßige klinische Anwendung der numerischen Flusssimulation derzeit jedoch nicht möglich. Zudem sind die quantitativen Daten der Flusssimulation durch die Vielzahl an Approximationen und Annahmen in ihrer Genauigkeit limitiert. Dennoch lassen sich bereits jetzt mit Hilfe der numerischen Flusssimulation qualitativ wertvolle Informationen, die über die reine Morphologie der Gefäße hinausgehen, ableiten. So können z. B. Verlaufsbeobachtungen bzgl. der Veränderung von Wanddrücken, Scherkräften und des Strömungsmustern mögliche Indikatoren für die Risikoabschätzung einer Ruptur oder einer Stentmigration bzw. Dislokation sein. Diese Indikatoren müssen in Zukunft im Rahmen prospektiver Verlaufsstudien an großen Patientenkollektiven definiert werden.

\section{Korrespondenzadresse \\ Dr. T. Frauenfelder}

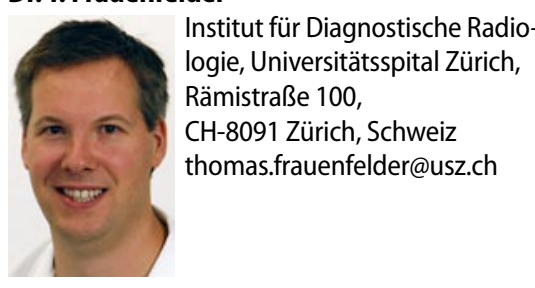

Interessenkonflikt. Der korrespondierende Autor gibt an, dass kein Interessenkonflikt besteht.

\section{Literatur}

1. Alcorn HG, Wolfson SK jr, Sutton-Tyrrell K et al. (1996) Risk factors for abdominal aortic aneurys$\mathrm{ms}$ in older adults enrolled in The Cardiovascular Health Study. Arterioscler Thromb Vasc Biol 16: 963-970

2. Newman AB, Arnold AM, Burke GL et al. (2001) Cardiovascular disease and mortality in older adults with small abdominal aortic aneurysms detected by ultrasonography: the cardiovascular health study. Ann Intern Med 134: 182-190

3. Di Martino ES, Guadagni G, Fumero A et al. (2001) Fluid-structure interaction within realistic three-dimensional models of the aneurysmatic aorta as a guidance to assess the risk of rupture of the aneurysm. Med Eng Phys 23: 647-655
4. Scott RA, Ashton HA, Lamparelli MJ et al. (1999) A 14-year experience with $6 \mathrm{~cm}$ as a criterion for surgical treatment of abdominal aortic aneurysm. $\mathrm{Br}$ J Surg 86: 1317-1321

5. Nicholls SC, Gardner JB, Meissner MH, Johansen HK (1998) Rupture in small abdominal aortic aneurysms. J Vasc Surg 28: 884-888

6. Valentine RJ, Decaprio JD, Castillo JM et al. (2000) Watchful waiting in cases of small abdominal aortic aneurysms-appropriate for all patients? J Vasc Surg 32: 441-448; discussion 448-450

7. Fillinger MF, Raghavan ML, Marra SP et al. (2002) In vivo analysis of mechanical wall stress and abdominal aortic aneurysm rupture risk. J Vasc Surg 36: 589-597

8. Finol EA, Keyhani K, Amon CH (2003) The effect of asymmetry in abdominal aortic aneurysms under physiologically realistic pulsatile flow conditions. J Biomech Eng 125: 207-217

9. Raghavan ML, Vorp DA, Federle MP et al. (2000) Wall stress distribution on three-dimensionally reconstructed models of human abdominal aortic aneurysm. J Vasc Surg 31: 760-769

10. Li Z, Kleinstreuer C (2005) Blood flow and structure interactions in a stented abdominal aortic aneurysm model. Med Eng Phys 27: 369-382

11. Li Z, Kleinstreuer C (2005) Fluid-structure interaction effects on sac-blood pressure and wall stress in a stented aneurysm. J Biomech Eng 127:662671

12. Liffman $K$, Lawrence-Brown MM, Semmens JB et al. (2001) Analytical modeling and numerical simulation of forces in an endoluminal graft. J Endovasc Ther 8: 358-371

13. Mohan IV, Harris PL, van Marrewijk CJ et al. (2002) Factors and forces influencing stent-graft migration after endovascular aortic aneurysm repair. J Endovasc Ther 9: 748-755

14. Frauenfelder T, Lotfey $M$, Boehm T, Wildermuth $S$ (2006) Computational fluid dynamics: hemodynamic changes in abdominal aortic aneurysm after stent-graft implantation. Cardiovasc Intervent Radiol 29: 613-623

15. Anderson JD (2006) Computational fluid dynamics. The basics with applications, 6th edn. McGraw Hill, New York

16. Long Q, Xu XY, Bourne M, Griffith TM (2000) Numerical study of blood flow in an anatomically realistic aorto-iliac bifurcation generated from MRI data. Magn Reson Med 43: 565-576

17. Lorensen WE, Cline HE (1987) Marching cubes: a high-resolution 3D surface construction algorithm. Comput Graph 21: 163-169

18. Scotti CM, Shkolnik AD, Muluk SC, Finol EA (2005) Fluid-structure interaction in abdominal aortic aneurysms: effects of asymmetry and wall thickness. Biomed Eng Online 4: 64

19. Scotti CMF, Finol EA (2007) Compliant biomechanics of abdominal aortic aneurysms: a fluid-structure interaction study. Comput Struct 85: 10971113

20. Bonert M, Leask RL, Butany J et al. (2003) The relationship between wall shear stress distributions and intimal thickening in the human abdominal aorta. Biomed Eng Online 2: 18

21. Simao da Silva E, Rodrigues AJ, Magalhaes Castro de Tolosa E et al. (2000) Morphology and diameter of infrarenal aortic aneurysms: a prospective autopsy study. Cardiovasc Surg 8: 526-532

22. Khanafer KM, Gadhoke P, Berguer R, Bull JL (2006) Modeling pulsatile flow in aortic aneurysms: effect of non-Newtonian properties of blood. Biorheology 43 : 661-679
23. Zarins CK, Bloch DA, Crabtree T et al. (2003) Stent graft migration after endovascular aneurysm repair: importance of proximal fixation. J Vasc Surg 38: 1264-1272; discussion 1272

24. Resch T, Malina M, Lindblad B et al. (2000) The impact of stent design on proximal stent-graft fixation in the abdominal aorta: an experimental study. Eur J Vasc Endovasc Surg 20: 190-195

25. Howell BA, Kim T, Cheer A et al. (2007) Computational fluid dynamics within bifurcated abdominal aortic stent-grafts. J Endovasc Ther 14: 138-143

26. Sternbergh WC 3rd, Money SR, Greenberg RK, Chuter TA (2004) Influence of endograft oversizing on device migration, endoleak, aneurysm shrinkage, and aortic neck dilation: results from the Zenith Multicenter Trial. J Vasc Surg 39: 20-26

27. Juchems MS, Pless D, Fleiter TR et al. (2004) [Noninvasive, multi detector row (MDR) CT based computational fluid dynamics (CFD) analysis of hemodynamics in infrarenal abdominal aortic aneurysm (AAA) before and after endovascular repair]. Rofo Fortschr Geb Rontgenstr Neuen Bildgeb Verfahr 176: 56-61

28. Li Z, Kleinstreuer C (2006) Computational analysis of type II endoleaks in a stented abdominal aortic aneurysm model. J Biomech 39: 2573-2582

29. Egelhoff CJ, Budwig RS, Elger DF et al. (1999) Model studies of the flow in abdominal aortic aneurysms during resting and exercise conditions. J Biomech 32: 1319-1329 